\title{
Die strafrechtliche Aufarbeitung von staatlich gesteuertem Unrecht in Ungarn
}

Von Unterstaatssekretär Dr. Károly Bárd, Ministerium der Justiz, Budapest

1.

Einige Wochen vor den zweiten freien Wahlen in Ungarn ist es noch zu keiner einzigen Verurteilung wegen eines unter dem alten Regime begangenen politischen Verbrechens gekommen. Das wäre an sich nicht besonders bemerkenswert, wenn nicht alle Parteien der ehemaligen Opposition vor vier Jahren in ihren Wahlprogrammen versprochen hätten, die Täter des früheren totalitären Systems vor Gericht zu stellen.

Man könnte meinen, die Geschichte setze sich fort: In der Vergangenheit wenigstens in den letzten zwanzig Jahren des nun verschwundenen realen Sozialismus - galt Ungarn als die „lustigste Baracke des Lagers“; die Instruktionen aus Moskau wurden nur widerstrebend vollzogen, manche auch sabotiert, wobei man stets darauf achtete, die sozialistischen Bruderländer seiner Treue zu versichern. Man könnte den Eindruck haben, der Prozeß der Vergangenheitsbewältigung verlaufe (bzw. verlaufe nicht) in gleicher Weise: Man spricht recht viel darüber, aber eigentlich ist kaum etwas geschehen. Mit der gleichen Unwilligkeit, mit der der Sozialismus praktiziert wurde, vollzieht sich jetzt die rechtliche Aufarbeitung der „sozialistischen“ Vergangenheit.

Wenn dies auch etwas unheroisch klingen mag, so zeigt sich darin doch die einfache Tatsache, daß Ausmaß und Intensität der Vergangenheitsbewältigung letzten Endes von der zu bewältigenden Vergangenheit abhängen. Wenn man sich vor Augen hält, daß in den letzten 35 Jahren des autoritären Regimes keine massiven Menschenrechtsverletzungen festgestellt wurden und daß sich der sogenannte Systemwandel mit einer gewissen Kooperation der früheren Machthaber vollzogen hat, ist es nicht verwunderlich, daß man bei der strafrechtlichen Ahndung staatlich gesteuerten Unrechts in Ungarn eher behutsam vorgeht. Im übrigen entspricht die oben formulierte Aussage auch nicht ganz der Wahrheit, jedenfalls dann nicht, wenn man in die strafrechtliche Aufarbeitung der Vergangenheit auch Strategien oder Maßnahmen einbezieht, die keine strafrechtliche Verurteilung der Täter enthalten, aber doch mehr oder weniger mit dem Strafrecht und der Strafjustiz verbunden sind. 
II.

Die rechtliche Aufarbeitung staatlich gesteuerten Unrechts in den ehemaligen sog. sozialistischen Staaten (aber auch allgemein in totalitären Staaten) hat sich nach dem Sturz des alten Regimes in drei voneinander mehr oder weniger getrennten Bereichen vollzogen. (Abfolge und Unterschiede in der Intensität der "Wiedergutmachung “ in diesen drei Bereichen bedürften einer umfassenden vergleichenden Untersuchung, die vermutlich die jeweiligen Besonderheiten der heute eher als Einheit aufgefaßten totalitären Regimes deutlich machen würde.)

Der erste Bereich umfaßt die Maßnahmen zur Wiedergutmachung der unter dem Unrechtssystem zugefügten Verletzungen. Unserem Thema stehen diejenigen Maßnahmen am nächsten, durch die versucht wird, ungesetzliche Verurteilungen und Bestrafungen zu kompensieren. In diesem Bereich haben die ungarische Gesetzgebung, Regierung und Justiz einiges geleistet. $\mathrm{Zu}$ erwähnen sind Kompensationsmaßnahmen im Arbeits- und Sozialversicherungsrecht (vor allem Ergänzung der Rentenansprüche) für Personen, die zu Unrecht verurteilt worden waren und verschiedene Arten des Freiheitsentzugs (Internierung, Deportation, Zwangsarbeit in der Sowjetunion) oder den Verlust ihrer Ämter erlitten hatten ${ }^{1}$.

Bereits vor den ersten freien Wahlen begann man mit den Bemühungen um eine Rehabilitierung der gesetzwidrig verurteilten Personen. Zur Überprüfung individueller Entscheidungen eignete sich insbesondere das in den sozialistischen Ländern bekannte außerordentliche Rechtsmittel des "Einspruchs im Interesse der Gesetzlichkeit". Unter vage und großzügig formulierten Voraussetzungen konnte mit Hilfe dieses Rechtsbehelfs das Verfahren sowohl bei Gesetzesverletzung (Fehler bei der Rechtsanwendung) als auch bei „Unbegründetheit" (mangelhafte Tatsachenfeststellung) wieder aufgenommen werden, zugunsten des Verurteilten ohne zeitliche Begrenzung. Die Wiederaufnahme des rechtskräftig abgeschlossenen Verfahrens lag nach dem Gesetz allerdings im Ermessen des Generalstaatsanwalts bzw. des Präsidenten des Obersten Gerichts. Verbunden mit der relativen Unbestimmtheit der materiellen Voraussetzungen schuf dies die Gefahr einer selektiven Handhabung des Einspruchs, der schließlich im Jahre 1992 durch das Verfassungsgericht für verfassungswidrig erklärt und abgeschafft wurde.

1 Siehe z. B. Regierungsverordnung Nr. 93/1990 (21. 11. 1990) (Magyar Közlöny 116/ 1990); Regierungsverordnung Nr. 74/1991 (10.6. 1991) (Magyar Közlöny 62/1991); Regierungsverordnung Nr. 112/1991 (2.9.1991) (Magyar Közlöny 95/1991). 
Der Hauptmangel des Einspruchs im Zusammenhang mit der Wiedergutmachung von ungerechten Verurteilungen lag jedoch darin, daß er nicht dazu führen konnte, daß diejenigen Verurteilungen aufgehoben wurden, in denen das Verfahren rechtmäßig abgelaufen und auch der Tatbestand hinreichend aufgeklärt worden war, in denen die Verurteilung aber dennoch unannehmbar war, da schon der ihr zugrunde liegende Straftatbestand gegen rechtsethische Grundsätze verstieß.

Aus diesen Gründen war ein Eingreifen des Gesetzgebers notwendig. Zwischen 1989 und 1992 wurden drei sog. Nichtigkeitsgesetze erlassen. Das erste annullierte die Verurteilungen im Zusammenhang mit der Niederschlagung des Volksaufstandes von $1956^{2}$. Durch das zweite Gesetz von 1990 wurden Verurteilungen aufgehoben, die in den Jahren 1945 bis 1963 wegen Straftaten gegen die äußere und innere Sicherheit des Staates sowie wegen Straftaten im Zusammenhang mit der Planwirtschaft ergangen waren ${ }^{3}$. Schließlich annullierte das dritte Nichtigkeitsgesetz von 1992 Urteile, die zwischen 1963 und 1989 wegen politischer oder politisch motivierter Straftaten verhängt worden waren ${ }^{4}$. Da es sich bei den in den Nichtigkeitsgesetzen angeführten Straftaten um höchst unterschiedliche Fälle handeln konnte (die Tatbestände betrafen auch Rechtsgüter, die in einem Rechtsstaat gleicherweise schurzbedürftig sind), wurde der auch vom „sozialistischen“ Ungarn ratifizierte Internationale Pakt über den Schutz bürgerlicher und politischer Rechte als Bezugspunkt genommen; die Entscheidung darüber, ob der Angeklagte in dem jeweiligen konkreten Fall wegen der Ausübung eines Rechts verurteilt worden war, das in dem Pakt garantiert ist, oder ob das zugrundeliegende Verhalten auch in einem Rechtsstaat strafwürdig gewesen wäre, blieb den Gerichten überlassen ${ }^{5}$.

Die Nichtigkeitsgesetze enthielten keine Regelung über materielle Wiedergutmachung. Hierzu wurde ein besonderes Gesetz erlassen ${ }^{6}$, das für die Opfer rechtswidriger Tötung oder Freiheitsberaubung feste Entschädigungssummen bzw. Leibrenten vorsieht. Das Gesetz gewährt Entschädigung nicht nur für Freiheitsentzug als Folge eines gerichtlichen Urteils, son-

2 Gesetz Nr. XXXVU/1989 (Magyar Közlöny 78/1989).

3 Gesetz Nr. XXVI/1990 (Magyar Közlöny 29/1990).

4 Gesetz Nr. XII/1992 (Magyar Közlöny 26/1992).

5 Eine Verurteilung wegen Aufwiegelung “ konnte z. B. einerseits wegen der Äußerung von Kritik am kommunistischen System erfolgen, andererseits aber auch aufgrund einer legitimen Beschränkung der Meinungsfreiheit, z. B. dann, wenn der Täter zum Haß gegen eine Minderheit aufgestachelt hatte.

6 Gesetz Nr. XXXII/1992 (Magyar Közlöny 56/1992). 
dern auch für Kriegsgefangenschaft in der Sowjetunion (die als Zwangsarbeit eingestuft wird), für Frontdienst im $Z$ weiten Weltkrieg (davon war vor allem die jüdische Bevölkerung betroffen) sowie für weitere Formen der Freiheitsberaubung ohne gerichtliche Entscheidung. Während der Ausarbeitung des Gesetzes kam es zu Auseinandersetzungen über den Kreis der zu entschädigenden Menschenrechtsverletzungen und über das Ausmaß der Wiedergutmachung; hierbei wurden die Zwangsmaßnahmen während des Zweiten Weltkrieges in teilweise geschmackloser Weise verharmlost. Dies zeugt von den Schwierigkeiten, die entstehen, wenn es eine Regierung auf sich nimmt, nicht nur die von der gerade überwundenen Diktatur verübten Rechtsverletzungen wiedergutzumachen, sondern auch Entschädigung für solche Beeinträchtigungen zu gewähren, die ein früheres totalitäres System hervorgerufen hatte und deren Wiedergutmachung bislang versäumt worden war.

Den Entschädigungsgesetzen liegt die Hypothese zugrunde, daß Menschenrechtsverletzungen auch in einer Diktatur im Rahmen eines formellen Verfahrens erfolgen. Zwar sieht das Gesetz eine Entschädigung auch für den Fall vor, daß das Opfer nicht erst aufgrund eines rechtskräftigen Urteils hingerichtet, sondern schon während des Strafverfahrens unter Mitwirkung der Ermittlungsbehörden getötet wurde; in den Fällen, in denen staatliche Funktionsträger jemanden töteten, ohne daß ein formelles Strafverfahren eingeleitet worden war, besteht jedoch kein Anspruch auf Entschädigung. Damit bleiben gerade die Fälle brutalster Gewaltanwendung ohne jede Wiedergutmachung; solche Fälle kamen sowohl während des Zweiten Weltkrieges als auch in der darauf folgenden Zeit in großem Umfang vor. Man darf nicht verschweigen, daß bei der Ausgestaltung des Entschädigungsgesetzes auch finanzielle Gesichtspunkte eine Rolle gespielt haben. Auch heikle politische Fragen, wie zum Beispiel die Begründung und das Ausmaß der Schuld Ungarns an der Vernichtung von Juden während des Zweiten Weltkrieges, mußten beantwortet werden.

Die Strategie der Bewältigung durch Wiedergutmachung wurde also vor allem bei solchen Rechtsverletzungen angewandt, die auf einer rechtlichen Grundlage im Rahmen eines formellen Verfahrens erfolgten, die also im eigentlichen Sinne staatlich gesteuert waren. Bei solchen Rechtsverletzungen ist die individuelle Schuld einzelner Täter schwer feststellbar: Wie soll man die Schuld aufteilen zwischen den obersten Parteifunktionären, die die Idee entwickelten, die Planwirtschaft mit strafrechtlichen Mitteln zu stärken, den "Gesetzgebern", die dieser Idee servil zustimmten, und den Richtern, die die so entstandene unvernünftige oder sittenwidrige Strafnorm anwandten und 
einen Bauern wegen des Schlachtens seines eigenen Viehs verurteilten? Strafrechtliche Ahndung und Verurteilung einzelner Täter kommt dagegen dort in Betracht, wo den Taten keine rechtliche Regelung zugrunde lag oder wo der Täter mit roher Gewaltanwendung über das vom Recht Geforderte hinausging. Diese Unterscheidung kann jedoch lediglich als Grundregel gelten. Es kann durchaus auch Normen geben, die in krassem Widerspruch zu den elementarsten Anforderungen der Menschenrechte stehen und deren Anwendung nicht entschuldigt werden kann. Doch zeigt sich auch in der Beurteilung der NS-Justiz sowie der Justiz der DDR durch die deutsche Lehre und Rechtsprechung die Tendenz, die strafrechtliche Verfolgung auf die sog. Exzeßtaten zu beschränken.

III.

Im Zuge der zweiten Strategie der rechtlichen Aufarbeitung staatlich gesteuerten Unrechts hat das ungarische Parlament vor kurzem ein Gesetz über die "Disqualifikation" von Informanten der ehemaligen Staatspolizei verabschiedet $^{7}$. An diesem Gesetz wurde einige Kritik geübt. Man bezweifelte vor allem die Zuverlässigkeit der Unterlagen; außerdem wurde der Regierungskoalition vorgeworfen, sie wolle dadurch, daß sie den Gesetzesentwurf erst einige Wochen vor den Wahlen und nach langer Verzögerung präsentierte, die Abgeordneten bzw. die Kandidaten einschüchtern. Da die bereits gewählten Abgeordneten durch das Gesetz nicht betroffen waren, könnten Verdachtsmomente gegen sie nicht ausgeräumt werden.

Die Zielsetzung des Gesetzes ist im übrigen zu begrüßen: Es soll verhindern, daß Agenten der Staatssicherheitspolizei als Abgeordnete, Regierungsmitglieder, leitende Staatsbeamte, Richter oder Staatsanwälte tätig werden bzw. in ihren Ämtern bleiben. Das Gesetz sieht vor, daß die Betroffenen darüber benachrichtigt werden, daß sie auf einer Liste von Informanten der Staatspolizei stehen. Der Betroffene kann dann von seinem Amt zurücktreten; tut er dies nicht, kann seine Agententätigkeit öffentlich bekanntgemacht werden. Zuvor kann sich der Betroffene jedoch auch an ein Gericht wenden.

Rechtsstaatliche Bedenken nufen die vagen Formulierungen des Gesetzes hervor; außerdem steht nicht fest, ob die Erstreckung der Überprüfung auf die Leiter von Unternehmen und Banken sowie auf die Redakteure von Zeitungen und Zeitschriften der Verfassung entspricht ${ }^{8}$.

7 Gesetz Nr. XXIIJ/1994 (Magyar Közlöny 36/1994).

8 Nach dem Gesetz sollen die Überprüfungen durch zwei Ausschüsse mit je drei Richtern 
IV.

Auch auf dem dritten Gebiet der Vergangenheitsbewältigung, bei der Feststellung der Verantwortlichkeit für die im früheren Regime begangenen Straftaten, wurde vieles versucht, doch sind die Ergebnisse bisher mager. Bevor ich auf sie eingehe, möchte ich bemerken, daß eine Verbindung zwischen den drei genannten Strategien (Wiedergutmachung, Disqualifikation, Bestrafung) besteht. Denn gerade die Kompensation der Opfer wirft für die Bevölkerung die Frage auf, wer die Verantwortung für das Unrecht, für das jetzt entschädigt wird, trägt. Auch der Gesetzgeber scheint sich diesem gedanklichen Zusammenhang nicht entziehen zu können. So enthält Art. 29 des tschechoslowakischen Gesetzes $117 / 1991$ über die gerichtliche Rehabilitation die Regelung, daß Straftaten, die im Zuge des Rehabilitationsverfahrens bekannt werden, noch bis 1995 verfolgt werden können, wenn nicht bereits zuvor die Verjährung eingetreten war. Eine etwas andersartige Verknüpfung zwischen den beiden Bereichen findet sich in dem ungarischen Gesetz über die Entschädigung der Opfer von Tötungen und Freiheitsberaubungen: Danach entfällt ein Entschädigungsanspruch, wenn der Anspruchsteller selbst die Menschenrechte anderer schwer verletzt hatte ${ }^{9}$. Eine solche Verknüpfung der Strategien der Vergangenheitsbewältigung läßt sich auch in Deutschland feststellen und ist auch dort nicht ohne Probleme ${ }^{10}$.

Was die Feststellung der Verantwortlichkeit der Täter betrifft, so lassen sich wiederum verschiedene Strategien unterscheiden, die überwiegend als gegensätzliche, teilweise auch als einander ergänzende Lösungen verstanden werden.

Die Einrichtung parlamentarischer Untersuchungsausschüsse ist auch in Ungarn diskutiert worden. Bereits im Herbst 1990 wurde dem Parlament ein entsprechender Gesetzesentwurf vorgelegt. Der dort vorgesehene Ausschuß hätte vornehmlich die politische Verantwortlichkeit der führenden Persönlichkeiten des früheren Regimes untersuchen sollen. Dieser Entwurf wurde nur ein einziges $\mathrm{Mal}$ im Verfassungs- und Justizausschuß des Parlaments be-

durchgeführt werden. Bislang konnte erst ein Ausschuß gebildet werden. Deshalb konnten noch keine Überprüfungen durchgeführt werden, obwohl das Gesetz bereits am 1.7. 1994 in Kraft getreten ist. In zahlreichen Anträgen beim Verfassungsgericht wird die Verfassungsmäßigkeit des Gesetzes in Zweifel gezogen.

Diese Bestimmung wift die interessante theoretische Frage auf, ob Opfer von Menschenrechtsverletzungen ihren Entschädigungsanspruch durch späteres Verhalten verwirken können.

10 Siehe hierzu Lüderssen, ZStW 104 (1992), S. 770. 
raten und geriet dann in Vergessenheit. Auch spätere Anläufe, als Alternative zur strafrechtlichen Ahndung parlamentarische Untersuchungsausschüsse einzurichten, blieben ohne Erfolg. Die Art und Weise, in der diese Vorschläge behandelt wurden, erweckt den Eindruck, daß die Einrichtung solcher Ausschüsse gar nicht ernsthaft erwogen worden ist. Dasselbe gilt für sog. Tribunale, deren mögliche Rolle bei einer Bewältigung der Vergangenheit in Ungarn - im Gegensatz zu Deutschland - nicht einmal diskutiert wurde. Dies verwundert allerdings nicht, denn in Ungarn wurde auch in der Ära des "Sozialismus" eine „Vergesellschaftung“ der Justiz, wie sie sich etwa in der DDR in Gestalt der gesellschaftlichen Gerichte zeigte, stets abgelehnt. Zwar wurden auch in Ungarn im Jahre 1962 entsprechend dem Plan Chruschtschows zur Vergesellschaftung der Rechtspflege gesellschaftliche Gerichte eingerichtet; sie haben aber nie richtig funktioniert, 1975 wurde ihnen die Zuständigkeit für Strafsachen entzogen, und danach sind sie ohne gesetzgeberisches Eingreifen ausgestorben. Auch weiterhin scheint man in Ungarn geneigt zu sein, die Aburteilung von Strafsachen allein der formellen Justiz zu überlassen.

\section{V.}

Das zentrale Problem bei den Versuchen zur Ahndung der unter dem früheren Regime begangenen Straftaten war die Verjährung. Dies ist nicht weiter erstaunlich, da es in den letzten 35 Jahren des sozialistischen Regimes in Ungarn nicht mehr zu massiven Menschenrechtsverletzungen gekommen ist. Es ist jedoch bemerkenswert, daß es auch zu keiner Verurteilung wegen nicht verjährter Delikte kam, obwohl Grund zu der Annahme besteht, daß sich die Funktionäre des früheren Regimes der Strafverfolgung durch eine Art Immunität entzogen hatten. So erließ das Politbüro der KP zuletzt noch im Jahre 1984 einen Beschluß, demzufolge Strafverfahren gegen die höchsten Funktionäre der Partei nur mit Zustimmung der entsprechenden Parteiorganisationen eingeleitet werden konnten. Über das konkrete Vorgehen in solchen Fällen bestimmten Instruktionen des Generalstaatsanwalts. Im übrigen hätte man die noch aus der kommunistischen Ära stammenden Straftatbestände gerade wegen ihrer vagen, den Grundsätzen der Bestimmtheit und der Rechtsstaatlichkeit widersprechenden Formulierung leicht anwenden können. Dies hätte jedoch das grundlegende ethische Dilemma der „Vergangenheitsbewältrigung“ aufgeworfen: Können Taten, die unter nicht rechtsstaatlichen Umständen bewirkt worden sind, überhaupt mit rechtsstaatlichen Mitteln bewältigt werden? Wenn man dies verneint, so stellt sich die 
weitere Frage, ob es sich ein Rechtsstaat erlauben kann, bei der Aufarbeitung der Vergangenheit unter Leugnung seiner eigenen Werte unrechtsstaatliche Mittel anzuwenden, oder ob er es hinnehmen muß, daß dic Täter des früheren Regimes ohne Folgen davonkommen. Diese Grundfragen sind im Verlaufe der Diskussion über die Aufhebung der Verjährung deutlich zutage getreten. Die Auseinandersetzung konzentrierte sich auf die Frage, ob die materielle Gerechtigkeit oder das formelle Rechtsstaatsprinzip Vorrang erhalten sollte.

Am 4. 11.1991 verabschiedete das Parlament auf einen Antrag zweier Abgeordneter der Regierungskoalition ein Gesetz, das nach Inhalt und Wortlaut weitgehend dem deutschen Gesetz über die Verjährung von SED-Unrechtstaten und dem tschechischen Gesetz von 1993 über die Gesetzwidrigkeiten des kommunistischen Regimes und den Widerstand gegen dieses Regime entspricht. Nach dem Gesetz sollte die Verjährungsfrist für die Straftaten des Landesverrats, der vorsätzlichen Tötung und der Körperverletzung mit Todesfolge, die zwischen dem 21. 12. 1944 und dem 2. 5. 1990 begangen wurden, von neuem beginnen, wenn der Staat seinen Strafanspruch aus politischen Gründen nicht geltend gemacht hatte. Damit wurde nicht nur die Verlängerung der gesetzlichen Verjährungsfrist für noch nicht verjährte Straftaten bezweckt, sondern es sollte auch die Verfolgung bereits verjährter Taten ermöglicht werden. Das ungarische Verfassungsgericht erklärte dieses Gesetz im März 1992 für verfassungswidrig; es verletze „jene Forderung des verfassungsmäßigen Strafrechts, nach der auf die Verjährung der Strafbarkeit von Straftaten - einschließlich der Unterbrechung und Hemmung der Verjährung - die bei der Begehung der Straftat geltenden Gesetze angewendet werden müssen, es sei denn, daß im Verjährungszeitraum für den Täter günstigere Regelungen in Kraft getreten sind ${ }^{\text {“11. }}$.

Im einzelnen traf das Verfassungsgericht folgende Feststellungen:

1. Es ist verfassungswidrig, bereits verjährte Straftaten erneut strafbar zu machen.

2. Die Verlängerung der gesetzlichen Verjährungsfrist für noch nicht verjährte Straftaten ist verfassungswidrig.

3. Die gesetzliche Unterbrechung der Verjährung noch nicht verjährter Straftaten ist verfassungswidrig. 
4. Die Bestimmung von Hemmungs- und Unterbrechungsgründen durch rückwirkendes Gesetz ist verfassungswidrig.

5. Hinsichtlich der Verjährung kann nach der Verfassung nicht danach unterschieden werden, ob der Staat seinen Strafanspruch aus politischen oder aus sonstigen Gründen nicht geltend gemacht hat.

6. Es verstößt wegen Unbestimmtheit gegen die Rechtssicherheit und ist deshalb verfassungswidrig, wenn der Umstand, daß „der Staat seinen Strafanspruch aus politischen Gründen nicht geltend gemacht hat", zu einem Grund für die Hemmung der Verjährung erklärt wird.

Aus den zitierten Feststellungen der Entscheidung geht eindeutig hervor, daß das Verfassungsgericht der formellen Rechtsstaatlichkeit die Priorität gegenüber der materiellen Gerechtigkeit einräumt. Der Rechtsstaat darf nach Meinung des Verfassungsgerichts auf Rechtsverletzungen nur in rechtsstaatlicher Weise reagieren. Niemandem dürften die rechtsstaatlichen Garantien verwehrt, selbst gerechte Forderungen nicht unter Mißachtung der rechtsstaatlichen Garantien durchgesetzt werden. Schließlich heißt es in der Entscheidung: „Gerechtigkeit und moralische Begründetheit können zwar Motive für die Strafwürdigkeit sein, jedoch muß die Rechtsgrundlage für die Strafbarkeit der Verfassung entsprechen."

Das Gericht lehnt also einen Neubeginn der Verjährung unter Berufung auf das Element der Rechtssicherheit innerhalb des Rechtsstaatsprinzips ab. Der Grundsatz der Rechtssicherheit erfordere, daß das Recht als Ganzes sowie jede einzelne Regel klar, eindeutig, in ihrer Auswirkung berechenbar und für die Adressaten strafrechtlicher Normen auch voraussehbar sind. Die Verjährungsregeln des Strafrechts, fährt das Gericht fort, gewährleisteten eine gesetzmäßige Strafverfolgung, indem sie der Ausübung der Strafgewalt zeitliche Grenzen setzten. Die Unterlassung der Strafverfolgung oder die Erfolglosigkeit von Ermittlungen gingen zu Lasten des Staates. Nach Eintritt der Verjährung stehe dem Täter der Ausschluß der Strafbarkeit als subjektives Recht zu.

Darüber hinaus verneint das Verfassungsgericht auch die Möglichkeit einer Verlängerung von noch nicht abgelaufenen Verjährungsfristen. Es erkennt zwar an, daß das Institut der Verjährung keine Garantie dafür bietet, $\mathrm{da} ß$ die Strafbarkeit innerhalb des im voraus festgelegten Zeitraums auch tatsächlich erlischt. Der Täter habe also kein subjektives Recht darauf, daß die Strafbarkeit innerhalb der vorgesehenen Zeit verjährt. Das Institut der Verjährung enthalte jedoch die Zusicherung, daß sich die Regeln zur Berech- 
nung der Verjährung während des Laufs der Frist nicht zu Lasten des Täters änderten. Die Strafgewalt des Staates müsse also im Zeitpunkt der Aburteilung denselben Schranken unterworfen werden wie sie im Zeitpunkt der Tatbegehung bestanden.

In dieser Entscheidung legt das ungarische Verfassungsgericht den Begriff der Rechtsstaatlichkeit ersichtlich anders aus als das deutsche Bundesverfassungsgericht in seinem Verjährungsbeschluß aus dem Jahre $1969^{12}$, und es findet auch eine andere Lösung für den Konflikt zwischen Rechtssicherheit und materieller Gerechtigkeit. Das Bundesverfassungsgericht sieht bekanntlich sowohl die Rechtssicherheit als auch die materielle Gerechtigkeit als Bestandteile der Rechtsstaatlichkeit an. Die Lösung von Konflikten überläßt es der Entscheidung des Gesetzgebers, solange er diese ohne Willkür trifft. Außerdem unterscheidet das Bundesverfassungsgericht zwischen echter und unechter Rückwirkung. Echte Rückwirkung tritt nur dann ein, wenn der Gesetzgeber an bereits abgeschlossene Sachverhalte ungünstigere Folgen knüpft, als sie im Zeitpunkt der Verwirklichung dieser Sachverhalte vorhersehbar waren. Unechte Rückwirkung liegt vor, wenn das Gesetz eine nachträgliche Verschlechterung bezüglich nicht abgeschlossener Sachverhalte vorsieht. Hier gewährt das deutsche Bundesverfassungsgericht Vertrauensschutz, freilich nicht ohne Ausnahmen.

Dagegen gibt das ungarische Verfassungsgericht bei Konflikten zwischen Rechtssicherheit und materieller Gerechtigkeit eindeutig der Rechtssicherheit den Vorrang; diese wird als „grundlegender Bestandteil des Rechtsstaa-

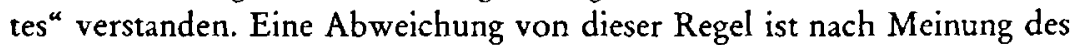
ungarischen Verfassungsgerichts nur dann zulässig, ,wenn dies aufgrund anderer, mit der Rechtssicherheit konkurrierender Verfassungsprinzipien als unvermeidlich erscheint und wenn hierdurch keine (im Vergleich zum angestrebten $Z$ weck) unverhältnismäßige Rechtsverletzung verursacht wird ... Das ungerechte Ergebnis bezüglich eines Rechtsverhältnisses ist jedoch an sich noch kein Argument gegen die Rechtssicherheit". Daraus ergibt sich, daß die Forderung nach materieller Gerechtigkeit nur innerhalb der Einrichtungen und Garantien durchgesetzt werden kann, die der Rechtssicherheit dienen sollen. Bereits früher hatte das Verfassungsgericht entschieden, daß es nach der ungarischen Verfassung kein subjektives Recht auf Durchsetzung der materiellen Gerechtigkeit gibt ${ }^{13}$.

13 Entscheidung 9/1992 (I. 30) AB (Magyar Közlöny 11/1992). 
Außerdem sieht das ungarische Verfassungsgericht die Bedeutung der Rechtssicherheit nicht aus dem Blickwinkel des Täters. Es kommt also nicht darauf an, ob dessen Erwartung legitim oder schützenswert ist. Das Gericht sieht das Problem vielmehr "von außen“, d.h. aus der Perspektive des Rechtssystems. Daher ist es verständlich, daß das Gericht den Unterschied zwischen echter und unechter Rückwirkung nicht als relevant ansieht und daß es folglich auch die Beseitigung des „kleinen“ Rückwirkungsverbots nicht zuläßt.

Es drängt sich die Frage auf, warum die beiden Verfassungsgerichte zu so unterschiedlichen Ergebnissen gelangt sind. Diese Differenz kann nicht durch unterschiedliche Formulierungen des jeweiligen Verfassungstextes erklärt werden. Vielmehr sollte man die Ursache in außerrechtlichen, nämlich in politischen und historischen Faktoren suchen. Das ungarische Verfassungsgericht konzediert ausdrücklich, daß seine Entscheidungen „historisch gebunden“ seien: „Auch dann, wenn das Verfassungsgericht absolute Werte verkündet, entdeckt es lediglich deren Sinn für sein Zeitalter."

Es läßt sich mit guten Gründen vermuten, daß es sich das deutsche Bundesverfassungsgericht im Jahre 1969 einfach nicht erlauben konnte, die Verlängerung der Verjährungsfrist für die bis dahin noch nicht verjährten NSVerbrechen zu beanstanden. Andererseits ist auch die Entscheidung des ungarischen Verfassungsgerichts mit der besonderen historischen Situation und vor allem mit dem Umstand zu erklären, daß der Systemwechsel in Ungarn - wie die Entscheidung hervorhebt - auf der Grundlage der Legalität erfolgt ist: „Die Verfassung und die fundamentalen Gesetze, die die - politisch gesehen - revolutionären Veränderungen eingeleitet haben, wurden unter Einhaltung der Rechtssetzungsnormen der alten Rechtsordnung und formell einwandfrei geschaffen, woraus sich auch ihre Verbindlichkeit ableitet." Daraus folgt für das ungarische Verfassungsgericht, daß erworbene Rechte geschützt bleiben müssen. Darüber hinaus müssen die Unantastbarkeit endgültig abgeschlossener Rechtsverhältnisse sowie die verfassungsmäßige Einschränkung der Abänderbarkeit solcher Dauerrechtsverhältnisse garantiert werden, die in der Vergangenheit entstanden sind: „Als wichtigste Regel folgt aus dem Prinzip der Rechtssicherheit, daß abgeschlossene Rechtsverhältnisse weder durch Rechtsnormen noch durch Außerkraftsetzung von Rechtsnormen verfassungsgemäß geändert werden können.“ 
VI.

Nach der zitierten Entscheidung des Verfassungsgerichts blieben zur Ahndung der unter dem alten Regime verübten Straftaten im wesentlichen zwei Wege offen: Man konnte versuchen, durch Gesetz ein sog. Feststellungsverfahren einzuführen, durch das die Schuld der Täter festgestellt und öffentlich bekanntgegeben wird, wenn auch auf eine Bestrafung verzichtet werden muß; oder man konnte versuchen, eine Lösung im Bereich der unverjährbaren Straftaten zu suchen.

Beide Wege sind beschritten worden. Am 16. 2. 1993 verabschiedete das ungarische Parlament ein Gesetz, das vorsah, daß das Verfahren bei Straftaten mit einer Strafdrohung von mehr als fünf Jahren Freiheitsstrafe erst nach gerichtlicher Verhandlung wegen Verjährung eingestellt werden kann. Dieses Gesetz verfolgte das Ziel, Strafverfahren wegen schwerer Taten bis zur öffentlichen Verhandlung zu bringen und die Schuld der im Ergebnis straffreien Täter wenigstens öffentlich bekanntzugeben. Wie zu erwarten war, erklärte das ungarische Verfassungsgericht auch dieses Gesetz für verfassungswidrig, und zwar mit der Begründung, es sei mißbräuchlich, das Instrumentarium des Strafverfahrens mit allen Möglichkeiten zur Einschränkung von Grundrechten auf solche Fälle anzuwenden, in denen diesen Grundrechtseinschränkungen jegliche verfassungsrechtlich und strafrechtlich legitime Zielsetzung fehlt ${ }^{14}$.

Die Regierung hat einen anderen Weg eingeschlagen. Ihr Gesetzesentwurf, der ebenfalls am 16. 2.1993 im Parlament verabschiedet wurde, schafft eigentlich kein neues Recht. Er stellt lediglich fest, daß die von Ungarn im Jahre 1954 ratifizierten Genfer Konventionen zum Schutze von Kriegsopfern im Jahre 1956 in Kraft waren, daß die dort angeführten schweren Rechtsverletzungen als Kriegsverbrechen bzw. als Verbrechen gegen die Menschlichkeit betrachtet werden müssen und daß sie deshalb - auch im Hinblick auf das durch Ungarn im Jahre 1971 ratifizierte Übereinkommen über die Nichtanwendbarkeit gesetzlicher Verjährungsfristen auf Kriegsverbrechen und Verbrechen gegen die Menschlichkeit - weiterhin verfolgbar sind. Durch dieses Gesetz wurde versucht, die Strafverfolgungsbehörden dazu zu veranlassen, die während des Freiheitskampfes von 1956 gegen die zivile Bevölkerung verübten Straftaten (vor allem die Schüsse auf Demonstranten) als unverjährbare Kriegsverbrechen bzw. Verbrechen gegen die Menschlichkeit zu ahnden. Zuvor hatten die Strafverfolgungsbehörden solche Verfahren mit der Begründung der Verjährung eingestellt. Die Staatsan- 
waltschaften hatten ausgeführt, daß die "schweren Verletzungen“ der Genfer Konventionen erst 1961 als unverjährbare Tatbestände in das ungarische Strafgesetzbuch aufgenommen worden seien. Im Jahre 1956 hätten sie also nicht als unverjährbare Tatbestände im ungarischen Recht existiert, so daß sie nicht mehr verfolgt werden könnten ${ }^{15}$. Die Staatsanwaltschaften gingen also davon aus, daß Völkerstrafrecht erst nach seiner Transformation in das nationale Recht von den ungarischen Gerichten angewandt werden könne.

Wiederum mußte das Verfassungsgericht entscheiden, das nunmehr das Verhältnis zwischen nationalem und internationalem Recht in einer in Ungarn bisher unbekannten Weise auslegte und das Gesetz für verfassungskonform erklärte ${ }^{16}$. Zunächst stellt das Verfassungsgericht fest, daß die Unverjährbarkeit nur in bezug auf solche Straftaten festgestellt werden kann, die bereits zur Zeit der Begehung nach ungarischem Recht unverjährbar waren, es sei denn, das Völkerrecht hätte das Verhalten als Kriegsverbrechen oder als Verbrechen gegen die Menschlichkeit eingestuft, seine Unverjährbarkeit gefordert oder zugelassen und für Ungarn hätte eine internationale Verpflichtung bestanden, die Verjährbarkeit auszuschließen. Zur Begründung beruft sich das Verfassungsgericht auf Art. 7 Abs. 2 EMRK und Art. 15 Abs. 2 IPBPR. Nach beiden Vorschriften ist die Verurteilung und Bestrafung einer Person nicht durch den Satz "nullum crimen sine lege“ ausgeschlossen, wenn die Tat im Zeitpunkt der Begehung nach den allgemeinen, von der Völkergemeinschaft anerkannten Rechtsgrundsätzen strafbar war. Dies ermöglicht die Verfolgung völkerrechtlicher Straftaten auch dann, wenn deren Tatbestand und Strafbarkeit im innerstaatlichen Strafrecht noch nicht normiert waren. Mit der Ratifikation des New Yorker Abkommens über die Unverjährbarkeit von Kriegsverbrechen im Jahre 1971 verpflichtete sich Ungarn, die Verjährung bei Kriegsverbrechen und bei Verbrechen gegen die Menschlichkeit (d.h. auch bei Taten, die die Genfer Konventionen als „schwere Verletzungen“ bezeichnen) auszuschließen. Die in dem New Yorker Abkommen aufgezählten Straftaten sind nach Auffassung des Verfassungsgerichts Teil des Völkergewohnheitsrechts. Sie zählen zu den von der Völkergemeinschaft anerkannten allgemeinen Rechtsgrundsätzen, zu den „allgemein anerkannten Regeln des Völkerrechts“, die nach der ungarischen Verfassung durch das ungarische Recht „anerkannt" werden. Aus dieser

15 Bis zum Inkrafttreten einer Novelle zum Strafgesetzbuch am 15. 5. 1993 (Gesetz XVII/ 1993) galten nur Kriegsverbrechen und Verbrechen gegen die Menschlichkeit als unverjährbare Straftaten. Durch die Novelle wurden auch qualifizierte Tötung und weitere schwere Verbrechen mit Todesfolge für unverjährbar erklärt.

16

Entscheidung 53/1993 (XI. 13) AB (Magyar Közlöny 147/1993). 
Formulierung zieht das Verfassungsgericht den Schluß, daß das Völkergewohnheitsrecht ohne besondere Transformation oder Übertragung zu den völkerrechtlichen Verpflichtungen zählt, deren Einklang mit dem innerstaatlichen Recht die Verfassung fordert. Adressaten des Völkergewohnheitsrechts seien nicht ausschließlich Staaten, sondern auch Individuen.

Hinsichtlich der Beziehung zwischen diesen Rechtssätzen des Völkergewohnheitsrechts und dem Grundsatz "nullum crimen sine lege“ führt das Gericht weiter aus, daß die internationale Regelung über Kriegsverbrechen und Verbrechen gegen die Menschlichkeit die Bestrafung dieser Straftaten unabhängig davon vorschreibe, ob sie im Zeitpunkt der Begehung nach innerstaatlichem Recht strafbar waren. Das Völkerrecht nehme also keine Rücksicht auf den Grundsatz „nullum crimen sine lege“ im innerstaatlichen Recht. Das Völkerrecht fordere lediglich, daß die Taten im Zeitpunkt ihrer Begehung durch die Völkergemeinschaft als Kriegsverbrechen oder Verbrechen gegen die Menschlichkeit angesehen worden sind.

Daraus zieht das Verfassungsgericht den Schluß, daß Kriegsverbrechen und Verbrechen gegen die Menschlichkeit in Ungarn auch dann verfolgt werden dürften, wenn das ungarische Recht zur Zeit der Begehung nicht über entsprechende Tatbestände verfügt hat. Es sei ausreichend, daß die Genfer Konventionen sowie das New Yorker Abkommen von Ungarn ratifiziert wurden. An diesem Ergebnis ändere auch die Tatsache nichts, daß der Text der Genfer Konventionen im ungarischen Gesetzblatt nie bekanntgegeben wurde, sondern daß dort lediglich die Tatsache der Ratifikation durch Ungarn vermerkt wurde. Da nämlich die Strafbarkeit von Kriegsverbrechen und Verbrechen gegen die Menschlichkeit unabhängig davon bestehe, ob sie mit dem Recht des Begehungsstaates übereinstimmt, sei es auch bedeutungslos, ob die Genfer Konventionen entsprechend den innerstaatlichen Regeln vor 1956 verkündet wurden. Denn die Verantwortlichkeit der Täter hänge lediglich vom Völkerrecht ab.

Diese Entscheidung des Verfassungsgerichts führte zu unterschiedlichen Reaktionen. Einige feierten sie als Sieg des humanitären Völkerstrafrechts, während andere heftige Kritik äußerten. Vor allem wurde beanstandet, daß das Gericht dem einzelnen gegenüber der mächtigen „internationalen $\mathrm{Ge}$ meinschaft" diejenigen Garantien entzogen habe, die ihm gegenüber dem innerstaatlichen Gesetzgeber zustehen ${ }^{17}$. Außerdem wurde die Frage aufge-

17 Bragyova, Historische Justiz und internationales Recht (ungarisch), Allam-és Jogtudomány 1993/3. 
worfen, ob die Verfahren gegen die Täter von 1956 die Funktion erfüllen könnten, über die Feststellung individueller Schuld hinaus in einem einwandfreien Prozeß die historische Wahrheit aufzuzeigen und ein moralisches Urteil zu fällen ${ }^{18}$.

Einige Verfahren wurden bereits eingeleitet, doch ist es bisher noch nicht zur Erhebung von Anklagen gekommen. Jedenfalls stehen die Justizorgane vor einer großen Aufgabe. Denn das vom Verfassungsgericht nunmehr für verfassungskonform erklärte Gesetz sagt lediglich, daß dann, wenn es in Ungarn nach dem 23. 10. 1956 einen bewaffneten Konflikt internationalen Charakters oder einen Bürgerkrieg gegeben haben sollte, die Tatbestände der "schweren Verletzungen“ im Sinne der Genfer Konventionen anzuwenden sind. Ob es jedoch einen Krieg gab oder ob man nur von einer „Intervention" sprechen kann, ob die Kriterien des Bürgerkrieges erfüllt waren und ob die Taten deshalb als Kriegsverbrechen oder als Verbrechen gegen die Menschlichkeit eingestuft werden können, dies alles müssen die Gerichte noch entscheiden.

18 Kis, Vor den Schießprozessen - Meditation über die Zeit (ungarisch), Kritika 1994, Heft 5. 